\title{
Feasibility of ultra-high-dimensional flow imaging for rapid pediatric cardiopulmonary MRI
}

\author{
Joseph Y Cheng ${ }^{1 *}$, Tao Zhang ${ }^{1}$, John M Pauly², Shreyas Vasanawala ${ }^{1}$ \\ From 19th Annual SCMR Scientific Sessions \\ Los Angeles, CA, USA. 27-30 January 2016
}

\begin{abstract}
Background
A comprehensive congenital cardiac MRI exam with contrast enhancement typically consists of multiple scans to evaluate dynamic contrast enhancement (DCE) characteristics, physiological function, blood flow, and anatomical assessment. However, multiple sequences require skilled operators, prolong the exam, and lengthen anesthesia duration for uncooperative patients. Thus, we propose a single comprehensive $5 D$ flow volumetric acquisition for a simpler and shorter cardiac exam. This technique resolves the cardiac cycle (1D), contrast dynamics (1D), and flow velocities for 3D volumes. The feasibility of the proposed $5 \mathrm{D}$ flow method is demonstrated for cardiopulmonary imaging.
\end{abstract}

\section{Methods}

Velocity-sensitization in 3D is integrated into a temporally resolved DCE sequence (T1-weighted RF-spoiled GRE) using 4 velocity-encoding echoes. The gradients for velocity encoding are also used to acquire intrinsic Butterfly navigators to monitor patient motion (JY Cheng et al ISMRM 2015, p451). During the acquisition with EKG monitoring, gadolinium contrast is intravenously administered. To enable compressed sensing, a pseudo-random $\mathrm{k}$ - $\mathrm{t}$ view- ordering scheme is used to acquire the $\mathrm{k}$-space data (JY Cheng et al JMRI 2015). Different sampling masks are applied during each velocity-encoding echo to achieve higher temporal resolution.

The data are binned into different volumes based on the cardiac phase, contrast phase, and velocity-encoding echo. This results in a 5D flow dataset. Though highly undersampled, the dataset can be reconstructed by exploiting data redundancies in multiple dimensions ( $M$ Lustig et al MRM 2007) and by applying parallel imaging (M Uecker et al MRM 2014). With the Butterfly motion estimates, soft-gating is used for suppressing motion artifacts (JY Cheng et al ISMRM 2015, p451). Spatial wavelet and total variation for the cardiac-cycle and contrast-phase dimensions are used for compressed-sensing regularization constraints (L Feng et al MRM 2013).

Two different reconstructions are performed to highlight different features: R1) DCE dynamics and R2) flow dynamics. For R1, fewer cardiac phases and more contrast phases are reconstructed to emphasize the DCE dynamics. For R2, more cardiac phases and a single contrast phase are reconstructed to characterize cardiac function and blood flow velocities.

\section{Results}

Angiography is depicted using R1 with a 2.1-s temporal resolution. By subtracting the baseline pre-contrast phases, the pulmonary perfusion dynamics can be characterized. The pulmonary vessels were first enhanced at $4.1 \mathrm{~s}$ and then at $6.1 \mathrm{~s}$ post-injection. Lastly, in R2, pulmonary and aortic flow can be quantified and visualized with velocity vector renderings.

\section{Conclusions}

The feasibility of a 5D flow technique has been demonstrated to characterize contrast-enhancement dynamics and blood flow. The integrated sequence has potential to simplify the acquisition process, enable advanced high-dimensional reconstructions, and improve postprocessing analysis.

\section{Funding:}

NIH R01-EB009690, NIH P41-EB015891, NIH R01EB019241, and GE Healthcare. 


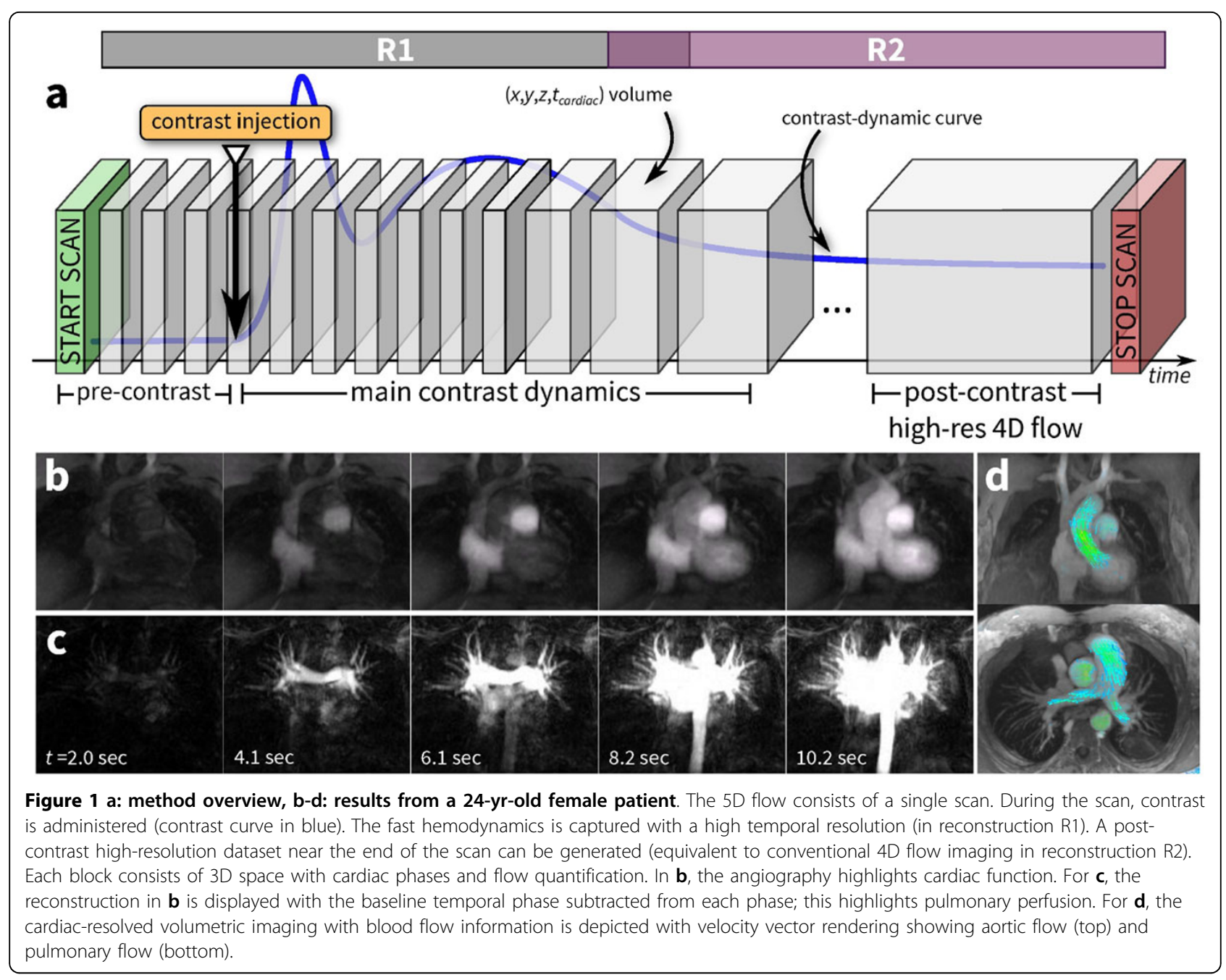

\section{Authors' details}

${ }^{1}$ Radiology, Stanford University, Stanford, CA, USA. ${ }^{2}$ Electrical Engineering, Stanford University, Stanford, CA, USA.

Published: 27 January 2016

doi:10.1186/1532-429X-18-S1-P217

Cite this article as: Cheng et al:: Feasibility of ultra-high-dimensional

flow imaging for rapid pediatric cardiopulmonary MRI. Journal of

Cardiovascular Magnetic Resonance 2016 18(Suppl 1):P217.

\section{Submit your next manuscript to BioMed Central} and take full advantage of:

- Convenient online submission

- Thorough peer review

- No space constraints or color figure charges

- Immediate publication on acceptance

- Inclusion in PubMed, CAS, Scopus and Google Scholar

- Research which is freely available for redistribution 